\title{
A Cloud Computing Based Mobile Census of Population and Housing System, Case of Central Statistics Office in Zambia
}

\author{
Article by Barbara Moto \\ Information Technology, Texila American University \\ E-mail: bmuyabi@gmail.com
}

\begin{abstract}
This research is a study of how census can be automated using hand held mobile devices. This automation may reduce on the possible and actual errors in the final processed information.

According to Shao, D. (2012) "The growing use of mobile technologies has increased pressure on the demand for mobile-based data collection solutions to bridge the information gaps for researchers" [1]. The law and technology favor an increased use of Information Technology platforms for data communications. Recently more bandwidth has become more available and cheaper and data transmission speed are faster with the launch of technologies such as $4.5 \mathrm{G}$ by Zamtel.

The objective of this study is to develop and implement a Cloud Computing Based Mobile Census of Population and Housing System using cloud computing technology and Geographic Information that addresses the current traditional way of Census Data Collection System. We will explain cloud computing can address problems related to efficiency and quality of statistical information for decision making. Secondly, we discuss the challenges that are anticipated in the implementation of Mobile Census of Population and Housing System. Thirdly we will discuss some of the areas of improvement expected in the implementation process of the Mobile Census of Population and Housing System for the Central Statistical Office. Fourthly we discuss the suitable technologies are available to support the development of the system. Finally, we will focus on the strategic advantages of developing a Cloud Computing Based Mobile Census of Population and Housing System and its security.
\end{abstract}

Keywords: Data Collection, Enumerator, Cloud Computing technologies, Zamtel, Census and CAPI.

\section{Introduction}

Census essentially refers to a complete counting all elements in a population. This process includes the collection, processing, compiling, evaluating, analyzing of collected data. It also includes the actual disseminating all census information for decision making. [3] This process may be automated or manual. In today's world, information technology affects and influences every aspect of human activity. The use of information technology to deploy automation may reduce on the time and cost spent on such an activity and improve on the efficiency and data integrity of the final processed information. This research is a study of how census can be automated using hand held mobile devices and the cloud for information processing and storage.

\section{The use of cloud computing technology}

Cloud computing is widely accepted to refer to remote data centers that host huge amounts networked servers which act as a central repository of for data [4]. This repository or data center provides various kinds of web services. Cloud computing when integrated into a mobile environment overcomes obstacles related to the performance, scalability and security. Cloud computing framework can be divided into Core services, Management and User interfaces. Each of these layers perform specific roles to contribute to the overall performance of the cloud to make available data storage and speedy data retrieval services to numerous users concurrently.

Cloud computing emanates from telecommunications where providers use virtual private network services for data communications. It relates to the computation, software, and data access and storage services. This does not need end-user knowledge of the actual physical location and server or technology configuration of the platform that is delivering the cloud services to the users.

Another study reinforced the need for an efficient land use planning, and represented the first 
approach integrating satellite imagery with population census data for studying the human environment in the Caribbean [5]. This helps in the mapping by capturing images using the satellite. This expedited the mapping process in the Caribbean and can be simulated in Zambia for use by 2020. The integration of satellite imagery during population census would be beneficial for this project is essential for Zambia in the planning of 2020 census, field management, area allocation to data collectors and locating households during field work.

The processes put in place to provide an internet option to the Australian public at the 2006 Census, was briefly outlined in another work [6]. Australia opted to use the internet option to harness the benefits of technological advances and their falling costs. Technology makes it possible to collect data offline and only use internet when uploading data to the cloud and servers. In the case of Zambia, the proposed system would allow the Census exercise to take advantage of information technology.

\section{Statement of the problem}

The Central Statistical Office in Zambia is affected by many problems ranging from collecting, processing and interpreting census data effectively and efficiently before it can be disseminated for decision making.

In October 2010, Zambia conducted its fifth comprehensive census of population and housing using traditional paper and pencil methods of data collection. Data capturing for this census took over a year to complete. The actual data analysis took yet another year.

During data collection for the census, the CSO, employed a large enumerators' workforce and data entry clerks to collect and capture data using scanners. From this traditions data collection method, several problems are evident. The researcher attempts to propose solutions to some of these with this study. The problems include

i. The Pace of data collection and capturing determines the lag between data collection, analysis and its use.

ii. Difficulty to ensure data integrity when using manual data collection methods.

iii. Use of a large workforce increasing time and labour costs.

iv. High cost of printing research tools and user manuals

\section{Objectives of the study}

The main objective of this study is to develop and implement a mobile data collection system using cloud computing technology and Geographic Information Systems that addresses the current manual systems.

We will explain the How the Mobile Census of Population and Housing System can address problems related to efficiency and quality of statistical information for decision making. Secondly, we will cover the challenges are anticipated in the implementation of Mobile Census of Population and Housing System. Thirdly we will discuss some of the areas of improvement expected in the implementation process of the Mobile Census of Population and Housing System for the Central Statistical Office. Fourthly we discuss the suitable technologies that are available to support the development of the system. Finally, we will focus on the strategic advantages of developing a cloud computing mobile data collection system and its security.

\section{How cloud computing addresses problems related to quality data collection}

The Data entered incorrectly is of little use, therefore in order to make sure that quality data is collected the following has to be done:

\section{Data entry training}

Data reliability starts at the source. In our case it will start with the numerators. As Enumerators are collecting and capturing data in the system, they are bound to introduce errors that compromise analytical results meant to guide decision makers. Therefore, it is important to ensure that staff members with system access are properly trained on data entry and upload protocols. There are several steps to consider when training:

- Training should be intensive and interactive, evolving process in response to operational needs. 
- Enumerators manual with procedures on how to collect data should be readily available for reference.

- System administrators should assign correct level of access to Enumerators and Supervisors based on their training and role.

- Auditing processes ought to be place in order for people to follow as they are checking for any inaccurate information entered into the system.

\section{Validation and verification}

Validation and verification checks are two ways to examine that the keyed in data into the computer or mobile device is correct. This is done by writing logics in the application which will check the skip patterns, ranges and consistency.

\section{Real time monitoring}

Information on the quality of the survey data collected in the Cloud Computing Based Mobile interviews and the process of conducting the interviews has to be monitored throughout the data collection period. Monitored information includes missing data and completeness among others. These data are analyzed regularly to assess quality of the interviews and compliance with the quality control put in place. This information is send as fed back to the interviewers when to make corrections when necessary.

\section{Challenges anticipated in the implementation of Mobile Census of Population and housing system}

Despite the benefit of acquiring high-quality data, there are also challenges anticipated in implementing the cloud mobile system such as Capacity building, data transfer, Skills, hiring a qualified field staff, data security.

\section{Capacity building}

Cloud Computing Based Mobile Census of Population and Housing System is a new technology in Zambia therefore Central Statistical Office will require to do a lot of capacity building for staff and the data collectors who be involved in the 2020 Census. This process is very expensive because it will require to train a good number of staffs for a period of not less than 14 days. The training will attract participant allowances hence it is costly because staff will be trained on the new methodology of collecting data using a mobile device.

\section{Data transfer}

Lack of Internet infrastructure in the rural areas of the country can result not uploading data to the central server hence hindering substantial progress in data processing, analysis and disseminating of Census results to the users.

\section{Skills}

Lack of technological skills by staff in development of the system such as; Programming, Networking, collaboration tools, Server administration, Storage management, Data analysis and visualisation can lead to failure in implementing the system.

\section{Hiring a qualified field staff}

Census is a big exercise which requires a good number of data collectors. Most of the participants are School leavers who may not possess the skills in mobile data collection.

\section{Data security}

Moto B. states that "During mobile data collection the data is stored on the device. The challenge comes in when the device is lost, there is a risk of losing data or the data may end up in the hands of unauthorised people who may not be part of the program and can result in compromising with confidentiality". 
DOI: 10.21522/TIJAR.2014.SE.19.01.Art004

ISSN: $2520-3088$

\section{Areas of improvement expected in the implementation process of the mobile census of population and housing system}

Cloud computing increases efficiency, Cost and time of disseminating Census results is reduced

\section{Efficiency}

The systems will be automated therefore the speed of conducting interviews will be reduced, interviewer is automatically prompted with the correct questions to ask, data will be ready for analysis as it is entered in directly into an electronic form, data will also be presented automatically through visualization technology which does not require someone doing it manually.

\section{Reduction on cost}

Cloud computing will reduce costs at all levels of the census processes such as:

i. No printing of questionnaire

ii. No transport is required to deliver questionnaires

iii. Reduce on Allowances as number of data collectors is reduced

iv. No data warehouse is required to store questionnaires

\section{Time}

Automation will reduce time to complete tasks such as

i. The time of conducting interviews can be reduced

ii. Time for Analysis is reduced

iii. Time is reduced to disseminate data

\section{Technology availability}

The following are the suitable technologies that are available to support the development of the system:

\section{Skills}

In order for Statistical Offices to develop a good and working system for data collection Staff need to poses skills which are available such as; Programming, Networking, Collaboration tools, Server administration, Storage management, Data analysis and visualization.

\section{Internet}

Data collected using cloud computing requires to be synchronised to the Sever through the Internet Technologies which are available such as; Wireless, WIFI/WiMAX, Ethernet/fibre Mobile Network $(3 \mathrm{G} / 4 \mathrm{G})$ and Bluetooth

\section{Hardware}

The following are the suitable technologies under hardware that are available to support the development of the system

i. Smartphone

ii. Tablet/PDA

iii. Laptop

iv. Computer,

v. and Servers

\section{Security}

While their benefits using cloud computing to conducting Census and Surveys there is need to ensure that user convenience never undermines protection of valuable organization or users' information. In order to secure the system and data there are suitable technologies available such as; ID and Password, Encryption and Encapsulation, Data Access controls, User roles and privileges, Physical security (Access control) and Virtual Private Network links (VPN) 


\section{Strategic advantage}

Data collection using Cloud Computing technology can be said to focus on the potential benefits that may accrue to the exercise in terms of efficiency, accuracy, data integrity and the speed of performing the census. Benefits such as speed of collection and processing of data for decision making are important so that the data can be put to use before it becomes obsolete.

Many organizations are increasingly focusing on new ways of conducting surveys that focus on electronic data collection and analysis methods that have since replaced the traditional ways of data collection. Decision makers, statisticians and stakeholders need accurate and timely data in order to improve the quality of their decisions.

The ever-increasing use of mobile technologies has increased pressure on the demand for mobilebased data collection solutions to bridge the information gaps for researchers. This is even more possible with the explosion of information technologies in Zambia. The law and technology favour an increased use of Information Technology platforms for data communications. [7] Recently more bandwidth has become available and cheaper, data transmission speeds are also faster with the launch of technologies such as $4.5 \mathrm{G}$ in 2018 by Zamtel.[8]

\section{Conclusion}

As we are well aware of the main risks and challenges in implementing such a solution, we always keep in mind the fact not underestimate how much work it takes to properly implement a census, even with

the use of the latest technology (IT or ICT). Thus, our paper comforts us in knowing that this solution will make it possible for ONS to enumerate and present the census data meticulously with minimum hardship. A key ingredient also, is our ongoing consultation with the different users and producers of statistical information as well as our national technology partners, namely mobile operators, internet providers and IT manufacturers.

\section{Reference}

[1]. Davis, G.B. and Olson, M.H. (1985) Management Information Systems, Conceptual Foundations, Structure and Development, New York, McGraw Hill.

[2]. Martinuzzi S, et al. (2007), Land development, land use, and urban sprawl in Puerto Rico integrating remote sensing and population census data, Landscape and Urban Planning, Landscape and Urban Planning Volume 79, Issues 3 - 4, Pages 288-297.

[3]. Shao, D. (2012), https://muep.mau.se/bitstream/handle/2043/13936/Deo_Shao_Thesis.pdf.

[4]. Toby J. Velte, Ph.D., Robert Elsenpeter, (2010), New York, Cloud Computing: A Practical Approach McGraw-Hill Companies.

[5]. United Nation, (1958). This process may be automated or manual. In today's world, Information Principles and Recommendations for Population and Housing Censuses, Revision 2.

[6]. Weibel D, et al., (2008), Demographic and health surveillance of mobile pastoralists in Chad: integration of biometric fingerprint identification into a geographical information system, Geospatial Health Vol.3, Issue 1, pp. 113124.

[7]. Weibel D, et al., (2008), Demographic and health surveillance of mobile pastoralists in Chad: integration of biometric fingerprint identification into a geographical information system, Geospatial Health Vol.3, Issue 1, pp. 113124.

[8]. Yaluma (2013) Zambia Telecommunications Company (Zamtel)

http://www.lusakavoice.com/2013/02/28/zamtel-to-provide-high-speed-3g-4g-internet-at-unwto/ 